\title{
Management Decisions for Rheumatoid Arthritis After Tumor Necrosis Factor Alpha Antagonist-Induced Lupus-Like Syndrome
}

\author{
Alexa MEARA, Ruth CHAN, Matthew HUSA \\ The Ohio State University Wexner Medical Center, Rheumatology, Columbus, United States
}

Tumor necrosis factor alpha (TNF- $\alpha$ ) antagonistinduced lupus-like syndrome (TAILs) $^{1}$ is characterized by rashes, arthralgias, fever, and/or serositis along with positive lupus serologies temporally associated with initiation of TNF- $\alpha$ antagonist medications. ${ }^{2}$ The incidence of TAILs may be increasing as TNF- $\alpha$ antagonists are more widely utilized. The management of rheumatoid arthritis (RA) patients who develop TAILs and the safety of re-challenging these patients with other biologic therapies remain largely unknown and understudied.

A 40-year-old female patient presented with a bilateral, symmetric polyarticular small joint inflammatory arthritis, morning stiffness, elevated acute phase reactants, and positive rheumatoid factor with negative anti-cyclic citrullinated peptide testing consistent with a diagnosis of RA. Triple therapy (hydroxychloroquine $400 \mathrm{mg}$ daily, methotrexate $20 \mathrm{mg}$ weekly, and sulfasalazine $1000 \mathrm{mg}$ twice daily) was initiated, which improved but did not resolve her synovitis. Therapy was escalated to infliximab $3 \mathrm{mg} / \mathrm{kg}$ every six weeks along with continued subcutaneous methotrexate (20 mg/week). Two months after initiating infliximab, she developed a pruritic photo-distributed rash on her chest and upper extremities, including diffuse erythema and discrete papules on her bilateral cheeks. The rash progressed with development of erythema and bleeding over the anterior chest, lips, arms and face after an episode of prolonged sun exposure, and she sought out dermatologic and rheumatologic re-evaluation. Repeat serological analysis demonstrated a high titer positive antinuclear antibody with a low positive double stranded deoxyribonucleic acid serology (Table 1). Dermatologic evaluation concluded that our patient's rash was consistent with acute lupus dermatitis; the onset of which was associated with infliximab initiation and worsened by sun exposure. A clinical diagnosis of TAILs was made. TNF- $\alpha$ antagonist therapy was stopped. Therapy was then switched to abatacept and three months of $10 \mathrm{mg}$ daily oral prednisone was initiated as treatment for the rash. The rash resolved, and abatacept and methotrexate have since controlled her RA after steroid cessation.

Table 1. Labs at diagnosis of antagonist-induced lupus-like syndromes

\begin{tabular}{ll}
\hline Serological marker & Result \\
\hline Anti-nuclear antibody & $1: 640$ (previously \\
& $1: 160 @ \mathrm{RA}^{*}$ diagnosis) \\
Anti-SSA antibody & Positive \\
Anti-dsDNA antibody & $14 \mathrm{IU} / \mathrm{mL}$ \\
Anti-Smith antibody & Negative \\
Anti-Histone antibody & $1.9 \mathrm{U}$ \\
Anti-Chromatin antibody & Positive \\
C3 (80-178 mg/dL) & $85 \mathrm{mg} / \mathrm{dL}$ \\
C4 (12-42 mg/dL) & $13 \mathrm{mg} / \mathrm{dL}$ \\
\hline Anti-SSA antibody: Anti-Sjögren's-syndrome-related antigen A; \\
Anti-dsDNA: Anti-doublestranded deoxyribonucleic acid; RA: Rheumatoid \\
arthritis; C3: Complement component 3; C4: Complement component 4.
\end{tabular}


This is a case of TAILs induced by infliximab with resolution following medication cessation and administration of low dose corticosteroids. To the best of our knowledge, no current method is available to predict which RA patients are at greater risk for TAILs. Seroconversion of anti-nuclear antibody and double stranded deoxyribonucleic acid has been observed in patients exposed to TNF- $\alpha$ antagonists with an incidence of 25\% to $80 \%$ and $5 \%$ to $15 \%$, respectively. ${ }^{2-4}$ The frequency of antibody seroconversion does not correlate reliably with the incidence of TAILs. However, the development of clinical symptoms of TAILs (i.e. arthritis, serositis, rash) has been observed in conjunction with seroconversion of anti-nuclear antibody serologies, typically at high titers. ${ }^{1,3,5}$

The majority of the literature on TAILs has focused on management. ${ }^{2,3,6,7}$ As in our case, common current practice involves cessation of the offending TNF- $\alpha$ antagonist. In some cases, the addition of steroids is necessary depending on the severity of the clinical presentation. ${ }^{4}$ The challenge presented in this case is the management of RA following the recognition and resolution of TAILs. Specific guidelines are not available to address issues including the safety of re-challenging patients with another TNF- $\alpha$ antagonist or alternative therapy. In the current literature, we identified key factors including the extent of involvement of TAILs that may guide management. If a patient has organ dysfunction secondary to TAILs, such as pneumonitis, kidney or liver injury etc., we propose avoidance of future use of TNF- $\alpha$ antagonists in the absence of more detailed clinical trials assessing their re-initiation in this context. Case reports of mild TAILs manifestations (mild rashes and/or arthritis) demonstrate no recurrence when re-challenged with a different TNF- $\alpha$ antagonist. ${ }^{7}$ However, the patient may be understandably hesitant to reinitiate TNF- $\alpha$ antagonist therapy, as was the case with our patient. In such scenario, we recommend both vigilant monitoring for the re-appearance of TAILs regardless of medication choice for subsequent RA therapy, ${ }^{8}$ as well as patient education and shared decision-making regarding their therapeutic options. Well-defined clinical trials are necessary to define the risks of TAILs as well as the appropriate management of RA following TAILs. In the absence of these studies, physicians should use shared decision making in developing therapeutic plans for RA as well as remain vigilant for the occurrence of adverse reactions to TNF- $\alpha$ antagonists.

\section{Declaration of conflicting interests}

The authors declared no conflicts of interest with respect to the authorship and/or publication of this article.

\section{Funding}

The authors received no financial support for the research and/or authorship of this article.

\section{REFERENCES}

1. Williams EL, Gadola S, Edwards CJ. Anti-TNFinduced lupus. Rheumatology (Oxford) 2009;48:71620.

2. Costa MF, Said NR, Zimmermann B. Drug-induced lupus due to anti-tumor necrosis factor alpha agents. Semin Arthritis Rheum 2008;37:381-7.

3. Moulis G, Sommet A, Lapeyre-Mestre M, Montastruc $\mathrm{JL}$. Is the risk of tumour necrosis factor inhibitorinduced lupus or lupus-like syndrome the same with monoclonal antibodies and soluble receptor? A case/ non-case study in a nationwide pharmacovigilance database. Rheumatology (Oxford) 2014;53:1864-71.

4. Almoallim $\mathrm{H}, \mathrm{Al}$-Ghamdi $\mathrm{Y}$, Almaghrabi $\mathrm{H}$, Alyasi $\mathrm{O}$. Anti-Tumor Necrosis Factor- $\alpha$ Induced Systemic Lupus Erythematosus. Open Rheumatol J 2012;6:315-9.

5. Ramos-Casals M, Brito-Zerón P, Soto MJ, Cuadrado MJ, Khamashta MA. Autoimmune diseases induced by TNF-targeted therapies. Best Pract Res Clin Rheumatol 2008;22:847-61.

6. Ramos-Casals M, Brito-Zerón P, Soto MJ, Cuadrado MJ, Khamashta MA. Autoimmune diseases induced by TNF-targeted therapies. Best Pract Res Clin Rheumatol 2008;22:847-61.

7. Williams VL, Cohen PR. TNF alpha antagonistinduced lupus-like syndrome: report and review of the literature with implications for treatment with alternative TNF alpha antagonists. Int $\mathrm{J}$ Dermatol 2011;50:619-25.

8. Santiago T, Santiago MG, Rovisco J, Duarte C, Malcata A, da Silva JA. A case of infliximab-induced lupus in a patient with ankylosing spondylitis: is it safe switch to another anti-TNF- $\alpha$ agent? Clin Rheumatol 2013;32:1819-22. 